\title{
ÉPOCA DE NASCIMENTO NO CRESCIMENTO DE BEZERROS ABERDEEN ANGUS CRIADOS NO RIO GRANDE DO SUL E SUAS IMPLICAÇÕES NO MELHORAMENTO GENÉTICO ${ }^{1}$
}

\section{CALVING SEASON ON GROWTH OF ANGUS CALVES RAISED IN THE STATE OF RIO GRANDE DO SUL - BRAZIL AND ITS IMPLICATIONS ON GENETIC IMPROVEMENT}

\author{
Fernando Flores Cardoso ${ }^{2}$, Ricardo Alberto Cardellino ${ }^{3}$, Leonardo Talavera Campos ${ }^{4}$
}

RESUMO

Os objetivos deste trabalho foram determinar a existência de interação entre época de nascimento e os efeitos de sexo do bezerro (S), idade da vaca (IV) e idade do bezerro (ID), e estimar o efeito da época de nascimento sobre o crescimento dos bezerros do nascimento ao sobreano. Foram utilizados registros de peso à desmama (PD) de 40.915 animais Aberdeen Angus, criados no Rio Grande do Sul, nascidos na primavera e no outono, entre os anos de 1974 e 1997. Desses, 12.706 tinham pesagem ao nascer e, 22.448, peso ao sobreano (PS). As análises foram realizadas por meio de dois modelos: o primeiro contendo todos os termos de interação, com a finalidade de verificar a possível importância da interação entre os efeitos de IV, S e ID com época de nascimento sobre o PD, e o segundo com os efeitos aninhados dentro de época de nascimento, para comparar o crescimento dos bezerros de outono e primavera. A interação de época de nascimento foi significativa com $S(P<0,0001), I V(P<0,0001)$ e ID $(P<0,05)$. Fatores de correção para idade da vaca, idade e sexo do animal usados nos programas de melhoramento genético no sul do Brasil, devem ser estimados dentro de época de nascimento. $O$ ganho do nascimento à desmama e PD dos animais nascidos na primavera foram, respectivamente, $15,6 \%$ e 12,9\% maiores que os dos nascidos no outono. $O$ ganho pós-desmama foi $22,6 \%$ maior no outono, gerando um PS 4,2\% maior nesses animais, em comparação aos nascidos na primavera.

Palavras-chave: bovinos de corte, ganho de peso, idade da vaca, idade do bezerro, peso ao nascer, sexo do bezerro.

\section{SUMMARY}

The purpose of this study was to determine the existence of interaction between calving season and sex of calf
$(S)$, age of dam (AoD) and age of calf $(A C)$ and estimate the effect of calving season on growth of calves from birth to yearling age. Weaning weights (WW) from 40,915 Angus calves raised on 69 ranches located in the State of Rio Grande do Sul, southern Brazil, born in the Spring and Fall from 1974 to 1997, were used. The data set contained 12,706 birth weight and 22,448 yearling weight $(Y W)$ records. The analyses were made using two models: the first, including all the interaction terms, was set up to verify the effect of two by two interactions involving calving season and the effects of $S, A o D$ and $A C$, and the second with those effects nested within calving season, to compare pre-and post-weaning performance of Spring and Fall calves. The interactions of calving season with $S(P<0.0001), A o D(P<0.0001)$ and $A C$ $(P<0.05)$ were significant. Correction factors for age of dam, age and sex of calf, used in genetic improvement programs in southern Brazil, must be estimated within calving season. Adjusted weaning gain and $W W$ of Spring calves were, respectively, $15.6 \%$ e $12.9 \%$ higher than those from Fall calves. Post-weaning gain was $22.6 \%$ higher for Fall calves, making $Y W$ $4.2 \%$ higher in these animals, compared to those born in the Spring.

Key words: age of calf, age of dam, beef cattle, birth weight, sex of calf, weight gain.

\section{INTRODUÇÃO}

A pecuária de corte no Rio Grande do Sul é baseada na exploração das pastagens nativas em regime extensivo. A produção dessas pastagens apresenta uma variação sazonal durante o ano, com um período de maior produção de matéria verde na

\footnotetext{
${ }^{1}$ Parte da Dissertação apresentada pelo primeiro autor à Faculdade de Agronomia "Eliseu Maciel” (FAEM), Universidade Federal de Pelotas (UFPEL), para obtenção do título de Mestre em Ciências. Financiada pela Coordenação e Aperfeiçoamento Pessoal de EnsinoSuperior (CAPES).

${ }^{2}$ Médico Veterinário, MSc., Associação Nacional de Criadores "Herd Book Collares", Doutorando, 1205 Anthony Hall, Department of Animal Science, Michigan State University, East Lansing, MI, EUA. 48824. Bolsista da CAPES. E-mail: cardosof@ msu.edu.

${ }^{3}$ Engenheiro Agrônomo., PhD, Professor. do Departamento de Zootecnia FAEM/UFPEL. Bolsista do CNPq. Campus Universitário S/Nº $96010-900$. Pelotas/RS. E-mail: rcard@ufpel.tche.br. Autor para correspondência.

${ }^{4}$ Engenheiro Agrônomo, MSc., Associação Nacional de Criadores "Herd Book Collares".
} 
primavera/verão e uma queda abrupta da produção durante os meses de inverno, ficando as pastagens improdutivas de maio a agosto (ROVIRA, 1996; SALOMONI \& SILVEIRA, 1996). Através da escolha da época de nascimento, pode-se ajustar as necessidades nutricionais das vacas de cria e dos bezerros à variação da disponibilidade de forragem no decorrer do ano.

Existem, basicamente, duas épocas de parição no RS: primavera e outono. A parição na primavera é tradicionalmente a mais usada, pois possibilita adequar a estação de maior crescimento das pastagens com as maiores exigências das vacas. Entretanto, nos últimos anos, observa-se uma tendência, por parte dos criadores, de condução de acasalamentos durante os meses de inverno (junho e julho), para obtenção de partos no outono, antecipando a cobertura de novilhas e vacas vazias (SALOMONI \& SILVEIRA, 1996). As vacas no outono parem após o verão em excelente condição corporal, o que permite um melhor desenvolvimento fetal no terço final de gestação, gerando maior peso ao nascer. Na primavera, os bezerros são, em geral, mais leves ao nascer, entretanto encontram uma época favorável ao seu desenvolvimento no período pré-desmama e apresentam peso, aos 205 dias, maior que os bezerros de outono, que nascem mais pesados, mas têm menor ganho médio diário nos meses de inverno (BARCELOS \& LOBATO, 1992a; CARDELLINO \& CARDELLINO, 1984; PONS et al. 1989; MONTGOMERY \& DAVIS, 1987). O peso aos 365 dias dos animais de outono é maior do que o dos nascidos na primavera, uma vez que a primeira fase pós-desmama corresponde ao período primavera/verão. Entretanto, aos 550 dias de idade, os pesos dos bezerros nascidos na primavera são relativamente maiores do que os dos nascidos no outono, uma vez que essa pesagem corresponde a um período de maior disponibilidade alimentar para os animais de primavera (BARCELOS \& LOBATO, 1992b; MACHADO JUNIOR et al., 1999).

Os programas de melhoramento genético têm considerado o efeito de época de nascimento dentro do grupo contemporâneo, desconsiderando interações entre a época de nascimento e outros fatores como idade da vaca, sexo e idade do bezerro. (CAMPOS, 1993). A existência de interação entre idade da vaca e época de nascimento já foi relatada em estudos com dados provenientes do RS. CARDELLINO \& CARDELLINO (1984) afirmaram a necessidade de caracterizar esse efeito, propondo a possibilidade de utilizar diferentes correções para idade da vaca segundo a época do ano. Baseado em resultados de pesquisa com dados de desmama de bezerros no RS, FRIES (1984) reco- mendou que registros de outono e primavera fossem analisados separadamente e que alguns termos de interação entre época e outros efeitos como idade do bezerro fossem estudados.

Com base na existência de duas épocas de parição no RS, que apresentam condições de ambiente diferentes, os objetivos deste trabalho foram: determinar a existência de interação entre época de nascimento e os efeitos de idade da vaca, sexo e idade do bezerro e sua importância para o melhoramento genético, e determinar o efeito da época de nascimento sobre o crescimento de bezerros criados no RS, do nascimento ao sobreano.

\section{MATERIAL E MÉTODOS}

O trabalho foi desenvolvido utilizando-se dados de campo coletados rotineiramente pelo Programa de Melhoramento de Bovinos de Carne PROMEBO da Associação Nacional de Criadores "Herd Book Collares" - ANC, nos rebanhos da raça Aberdeen Angus que participaram do programa, durante os anos de 1974 a 1997. Foram utilizados os registros de desmama de 40.915 animais, dos quais 12.706 tinham pesagem ao nascer e 22.448 foram pesados ao sobreano.

Os animais foram criados em 69 propriedades localizadas no RS, em condições extensivas de pastagem. O clima da região é subtropical, com chuvas distribuídas durante o ano todo e uma precipitação média anual ao redor de $1.350 \mathrm{~mm}$. A temperatura média anual é de $17,6^{\circ} \mathrm{C}$, sendo, nos meses mais quentes, de $24^{\circ} \mathrm{C}$ e, nos mais frios, de $12,5^{\circ} \mathrm{C}$ (MORENO, 1961). A pastagem nativa é composta de gramíneas e algumas leguminosas, de crescimento basicamente estival. Algumas propriedades utilizam pastagens cultivadas durante os meses de inverno, compostas de aveia (Avena sp.), azevém (Lolium multiflorum sp.), cornichão (Lotus corniculatus L.) e trevos (Trifolium sp.). Os rebanhos de cria permanecem quase que exclusivamente em campo nativo. Os bezerros, após a desmama, algumas vezes, são manejados em pastagem cultivada, entretanto, em sua maioria, permanecem em campo nativo.

$\mathrm{O}$ peso ao nascer (PN) foi coletado nas primeiras 24 horas de vida do animal, em campo, normalmente com uma balança do tipo dinamômetro, no mesmo momento em que foram anotadas as informações de nascimento (data, número da mãe, número do bezerro, peso ao nascer e tipo de parto normal ou com dificuldade). Os animais foram pesados à desmama e ao sobreano. A pesagem de sobreano foi feita entre os 365 e 550 dias de vida, dependendo do nível alimentar do animais, sendo 
aqueles provenientes de regimes mais intensivos pesados mais jovens do que aqueles vindos de regimes mais extensivos. No outono, a pesagem dos animais ao sobreano foi feita normalmente aos 365 dias, antes do início do inverno. Além do peso à desmama (PD) e ao sobreano (PS), foram utilizados, nas análises, o ganho de peso ajustado para 205 dias (G205) e o ganho de peso ajustado para o período entre 205 e 410 dias de idade (G410). Os ajustes de ganhos de peso para idades padrões foram feitos segundo as normas da BEEF IMPROVEMENT FEDERATION (1996).

Os registros foram divididos em duas épocas de nascimento: outono, compreendendo os meses de fevereiro a junho e correspondendo a 6.141 registros ou 15\% dos nascimentos; e primavera, de julho a janeiro com 34.774 registros e representando $85 \%$ dos animais nascidos. A determinação do período correspondente a cada época de nascimento foi realizada com base nas freqüências de nascimentos determinadas pela data juliana do nascimento (Figura 1), caracterizando os dois sistemas de parição encontrados no sul do País, outono e primavera.

Os grupos contemporâneos foram formados incluindo o ano, criador, rebanho, grupo de manejo ou código alimentar e data da pesagem dos animais. Animais com mais de 120 dias de diferença de idade foram considerados em grupo contemporâneo à parte. Grupos contemporâneos com menos de dez animais e touros com menos de cinco filhos foram previamente excluídos da análise.

$\mathrm{O}$ efeito do mês de nascimento foi incluído no modelo para considerar variações ambientais que ocorrem dentro de época de nascimento. Os meses com menor número de nascimentos (dezembro-janeiro e maio-junho) foram agrupados e os meses com maior número de nascimento (abril, agosto, setembro, outubro e novembro) foram divididos em quinzenas, para permitir um melhor balan-

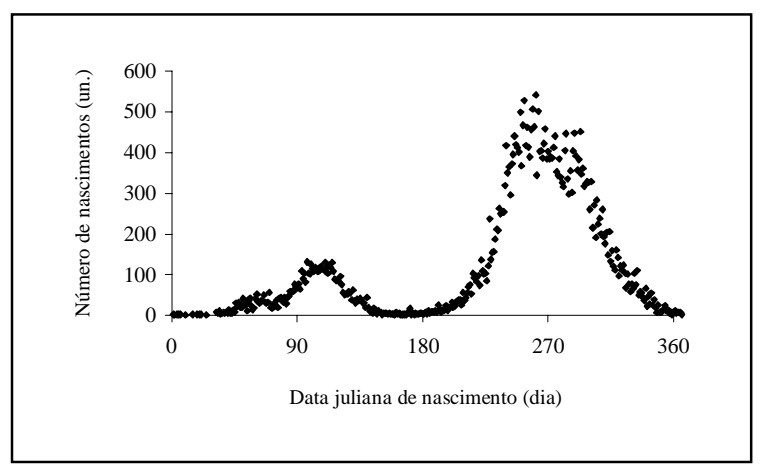

Figura 1 - Freqüência de nascimentos no decorrer do ano, de acordo com a data juliana de nascimento, de bezerros Aberdeen Angus criados no RS, entre os anos de 1974 e 1997. ço no número de informações nas subclasses e, conseqüentemente um melhor ajuste dos dados.

Dois modelos foram utilizados na análise dos dados. O modelo 1 foi constituído incluindo-se termos de interação entre época de nascimento e os efeitos de idade da vaca, sexo e idade do bezerro, e teve por objetivo verificar a existência e a importância dessas interações sobre o desempenho dos bezerros à desmama. Após verificar a importância desses efeitos para peso à desmama, o modelo 2 , apresentado a seguir, foi utilizado na comparação do desempenho de crescimento do nascimento ao sobreano, entre bezerros nascidos no outono e na primavera.

$$
\begin{aligned}
& \mathrm{y}_{\mathrm{ijkmnop}}=\mu+\mathrm{EP}_{\mathrm{i}}+\mathrm{GC}_{\mathrm{ij}}+\mathrm{M}_{\mathrm{ik}}+\mathrm{IV}_{\mathrm{im}}+\mathrm{S}_{\mathrm{in}}+\mathrm{IV}_{\mathrm{im}} * \mathrm{~S}_{\mathrm{in}}+ \\
& \mathrm{ID}\left(\mathrm{EP}_{\mathrm{i}}\right)+\mathrm{ID}^{2}\left(\mathrm{EP}_{\mathrm{i}}\right)+\mathrm{T}_{\mathrm{o}}+\mathrm{e}_{\mathrm{ijkmnop}}
\end{aligned}
$$

onde, $\mathrm{y}_{\mathrm{ijkmnop}}$ é o valor observado (PN, PD, G205, PS e $\mathrm{G} 410) ; \mu$ a média geral; $\mathrm{EP}_{\mathrm{i}}$ o efeito da época de nascimento (outono e primavera); $\mathrm{GC}_{\mathrm{ij}}$ o efeito do grupo contemporâneo dentro de EP (1-676); $\mathrm{M}_{\mathrm{ik}} \mathrm{O}$ efeito do mês de nascimento dentro de EP (1-15); $\mathrm{IV}_{\mathrm{im}}$ o efeito da idade da vaca dentro de EP (2-12 anos); $S_{\text {in }}$ o efeito do sexo dentro de EP (1-M e 2-F); $\mathrm{IV}_{\mathrm{im}} * \mathrm{~S}_{\mathrm{in}}$ o efeito da interação entre a idade da vaca e o sexo do bezerro dentro de $\mathrm{EP} ; \operatorname{ID}\left(\mathrm{EP}_{\mathrm{i}}\right)$ o coeficiente de regressão linear para idade do animal dentro de EP (100-300 dias); $\operatorname{ID}^{2}\left(\mathrm{EP}_{\mathrm{i}}\right)$ o coeficiente de regressão quadrático para idade do animal dentro de EP; $\mathrm{T}_{\mathrm{o}} \mathrm{o}$ efeito aleatório do touro, e $\mathrm{e}_{\mathrm{ijkmnop}} \mathrm{O}$ erro residual.

O modelo 2 foi uma simplificação do modelo 1 para a obtenção das estimativas dos efeitos fixos e médias de quadrados mínimos dentro de cada estação. Para a fase de nascimento foi suprimido, do modelo 2, o efeito de idade do animal. O mesmo modelo foi usado para a fase de sobreano. A análise dos dados foi realizada através do PROC MIXED do SAS - Statistical Analysis System (SAS INSTITUTE INC.,1996).

\section{RESULTADOS E DISCUSSÃO}

A análise de variância para $\mathrm{PD}$, pelo modelo 1, demonstrou a existência de interações altamente significativas $(\mathrm{P}<0,001)$ de época de nascimento com sexo do bezerro e idade da vaca. A interação foi significativa também para época e idade do bezerro $(\mathrm{P}<0,01)$. Esses resultados confirmam a hipótese sugerida em outras pesquisas com dados do RS, de que existe uma interação importante entre época de nascimento e outros efeitos de ambiente na 
fase de desmama (CARDELLINO \& CARDELLINO, 1984; FRIES, 1984) e indicam a necessidade de se estimar separadamente os ajustes para idade da vaca, sexo e idade do bezerro para cada época de nascimento. A partir dos resultados obtidos para PD nestes estudo, optou-se por analisar todas as características através de um modelo no qual todos os efeitos fixos foram considerados dentro de época de nascimento.

As médias ajustadas, obtidas através do modelo 2, para pesos e ganhos de peso dentro de cada época de nascimento (outono e primavera) são apresentadas na tabela 1. A idade média das vacas com parto no outono foi de 6,6 anos, sendo maior que na primavera (5,7 anos). Esses dados são semelhantes aos encontrados por BAGLEY et al. (1987) e são um indício de que, para as condições do RS, as vacas acasaladas para parirem no outono são aquelas que falham em conceber na primavera.

$\mathrm{O}$ PN médio mais alto na primavera $(\mathrm{Ta}-$ bela 1) está em desacordo com outros resultados de pesquisas no RS (BARCELOS \& LOBATO, 1992a) e em países de clima semelhante (MONTGOMERY \& DAVIS, 1987), entretanto, a diferença observada foi somente de $2 \%$ e, provavelmente, deve-se ao fato de o presente estudo incluir na primavera, os animais nascidos nos meses de dezembro e janeiro, quando são esperados pesos maiores ao nascimento. Outros autores não encontraram diferença significativa para peso ao nascer entre primavera e outono (BAGLEY et al., 1987).

O G205 e o PD dos animais nascidos na primavera são respectivamente $15,6 \%$ e $12,9 \%$ maiores que os dos nascidos no outono (Tabela 1), demonstrando que a primavera é altamente favorável ao crescimento dos animais até a desmama, pois os maiores ganhos de peso dos bezerros estão associa-

Tabela 1 - Medidas ajustadas e erros padrões, por épóca de nascimento, para pesos ao nascer, à desmama e ao sobreano e ganhos nascimento-desmama e pós-desmama, de bezerro Aberdeen Angus.

\begin{tabular}{lrr}
\hline & \multicolumn{2}{c}{ Médias ajustadas + erros padrão $^{\mathrm{a}}$} \\
\cline { 2 - 3 } & \multicolumn{2}{c}{} \\
\cline { 2 - 3 } Caráter & Outono & Primavera \\
& & \\
Peso ao nascer $(\mathrm{kg})$ & $32,0 \pm 0,27 \mathrm{a}$ & $32,7 \pm 0,10 \mathrm{~b}$ \\
Peso à desmama $(\mathrm{kg})$ & $141,8 \pm 0,55 \mathrm{a}$ & $160,2 \pm 0,35 \mathrm{~b}$ \\
Ganho nascimento-desmama $(\mathrm{kg})$ & $111,6 \pm 0,54 \mathrm{a}$ & $129,0 \pm 0,35 \mathrm{~b}$ \\
Peso ao sobreano $(\mathrm{kg})$ & $273,0 \pm 17,37 \mathrm{a}$ & $261,9 \pm 1,62 \mathrm{~b}$ \\
Ganho pós-desmama $(\mathrm{kg})$ & $86,3 \pm 9,30 \mathrm{a}$ & $70,4 \pm 0,86 \mathrm{~b}$
\end{tabular}

${ }^{a}$ Médias na mesma linha seguidas de letras diferentes diferem significativamente pelo teste $\mathrm{T}(\mathrm{P}<0,02)$. dos à maior produção da pastagem nativa. Esses resultados estão de acordo com os encontrados por outros autores (BARCELOS \& LOBATO, 1992a; CARDELLINO \& CARDELLINO, 1984; PONS et al. 1989; MONTGOMERY \& DAVIS, 1987), que constataram crescimento entre $15 \%$ e $23,2 \%$ maior nos bezerros de primavera.

O G410 dos animais de outono foi $22,6 \%$ maior do que o dos nascidos na primavera (Tabela 1), refletindo melhor condição alimentar no período pós-desmama. BARCELOS \& LOBATO (1992b), em um experimento em campo nativo no RS, encontram efeito altamente significativo da época de nascimento sobre o ganho pós-desmama. Entre 205 e 365 dias de idade, o maior ganho foi observado nos bezerros de outono, e entre 365 e 550 dias, nos animais de primavera. Resultados semelhantes foram encontrados por MACHADO JUNIOR et al. (1999), em um estudo sobre o crescimento de animais Brangus da EMBRAPA-CPPSUL. O maior G410 dos animais de outono, no presente estudo, fez com que esses animais atingissem praticamente o mesmo peso ajustado aos 410 dias daqueles da primavera, que foram consideravelmente mais pesados à desmama.

Em relação ao PS, calculado nesse trabalho para a idade média de 523 dias para todos os animais, os nascidos no outono foram $4,2 \%$ mais pesados (Tabela 1). Esse resultado, contudo, deve ser visto com restrição, pois existe uma diferença muito grande entre as médias de idade ao sobreano no outono (389 dias) e na primavera (556 dias). A diferença de idade entre os animais de outono e primavera ao sobreano torna inapropriado o ajuste do peso nessa fase, pela inclusão dos coeficientes de regressão linear e quadrático para idade no modelo estatístico.

O ganho médio diário observado do nascimento à desmama foi de $0,622 \mathrm{~kg}$ e $0,525 \mathrm{~kg}$, respectivamente, na primavera e no outono. Esses valores, embora estejam abaixo do potencial dos animais, estão num patamar razoável. Já os ganhos médios diários pós-desmama de $0,350 \mathrm{~kg}$ na primavera e de 0,383 no outono são bastante baixos, mesmo para sistemas extensivos, e evidencia que o regime nutricional desses rebanhos está aquém das exigências dos animais, mesmo para os animais de outono, que são recriados num período climático mais favorável.

O efeito da variação no ganho de peso, entre animais nascidos no outono ou na primavera, sobre a seleção, pode ser minimizado considerando-se a época de nascimento nas avaliações 
genéticas, utilizando-se fatores de correção estimados para cada época separadamente e, ainda, pela seleção dos animais dentro de época de nascimento. Um problema potencial é uma possível interação entre touro e época de nascimento, especialmente se determinados touros são usados somente no outono ou somente na primavera. Optar por uma ou outra época de nascimento, nos rebanhos que fazem melhoramento genético, é um decisão dos criadores e que envolve outros aspectos econômicos não estudados neste trabalho. Pode-se esperar grande concentração de nascimentos na primavera, mas com uma porção desses ocorrendo também no outono. Certamente, os programas de melhoramento precisam ter meios adequados (fatores de correção, modelos estatísticos) para solucionar os problemas referentes à estimação de valores genéticos, que possam ser comparáveis entre duas épocas de nascimento com marcadas diferenças ambientais.

\section{CONCLUSÕES}

É importante considerar a época de nascimento dos animais e sua interação com outros fatores de ambiente nas avaliações genéticas conduzidas pelo PROMEBO e por outros programas de melhoramento genético desenvolvidos no RS. Devem ser estimados fatores de correção para idade da vaca dentro de cada época de nascimento e os ajustes para idade e sexo do animal devem ser realizados considerando a época de nascimento.

Para os criadores especializados na produção de bezerros, a parição de primavera é a mais recomendável, do ponto de vista do crescimento prédesmama dos animais, medido pelo G205 ou pelo peso à desmama. Em relação ao G410 e ao PS, a decisão de optar por uma ou outra época de parição dependerá dos objetivos do criador, por exemplo, da época e idade que deseja fazer o primeiro acasalamento, da época e idade programada para o abate dos animais, bem como dos seus recursos alimentares para cada uma das épocas do ano.

O G410 é preferível em relação ao PS para avaliação conjunta do desempenho pósdesmama de animais nascidos na primavera e no outono, devido à diferença de idade que existe entre esses quando é feita a pesagem de sobreano. O G410 ajusta o ganho para uma idade média entre as idades mais jovens no outono e mais velhas na primavera, quando são realizadas as pesagens dessa fase.

\section{REFERÊNCIAS BIBLIOGRÁFICAS}

BAGLEY, C.P., CARPENTER Jr., C.J., FEAZEL J.I., $\boldsymbol{e} \boldsymbol{t} \boldsymbol{a l}$. Influence of calving season and stocking rate on beef cowcalf productivity. Jornal of Animal Science., v.64, p.687694, 1987.

BARCELOS, J.O.J., LOBATO, J.F.P. Efeitos da época de nascimento no desenvolvimento de bezerros Hereford e suas cruzas. I. Peso ao nascer e ganho médio diário pré-desmama. Revista da Sociedade Brasileira de Zootecnia, Viçosa, v.21, n.1, p.137-149, 1992a.

BARCELOS, J.O.J., LOBATO, J.F.P. Efeitos da época de nascimento no desenvolvimento de bezerros Hereford e suas cruzas. II. Peso ao desmame, ano e sobreano. Revista da Sociedade Brasileira de Zootecnia, Viçosa, v.21, n.1, p.150-157, 1992b.

BEEF IMPROVEMENT FEDERATION. Guidelines for uniform improvement programs. Raleigh : U. S. Dept. Agriculture - North Carolina State University, 1996. 155p.

CAMPOS, L.T. Material técnico de divulgação. Programa de melhoramento de bovinos de carne. Pelotas, RS : Associação Nacional de Criadores "Herd Book Collares", 1993. 26p. (Mimeografado).

CARDELLINO, M.V., CARDELLINO R.A. Efeitos ambientais sobre peso, ganho de peso e conformação à desmama em bovinos Hereford no Rio Grande do Sul. Revista da Sociedade Brasileira de Zootecnia, Viçosa, v.13, n.4, p.547-556, 1984.

FRIES, L.A. A study of weaning weights in Hereford cattle in the state of Rio Grande do Sul, Brazil. Ames, 1984. 317p. Thesis (Ph.D.) - Iowa State University, 1984.

MACHADO JUNIOR, P.C., SALOMONI, E. OSÓRIO, J.C.S Desenvolvimento ponderal de bovinos meio-sangue IbagéHereford nascidos em distintas estações do ano. Ciência Rural, Santa Maria, v.29, n.2, p.325-329, 1999.

MONTGOMERY, G.W., DAVIS, G.H. A comparison of spring and autumn calving for beef cattle production. In: NEW ZEALAND SOCIETY OF ANIMAL PRODUCTION, 47, 1987, Palmerston North. Proceedings... Palmerston North: NZSAP, 1987. p.115-118. 256p.

MORENO, J.A. Clima do Rio Grande do Sul. Porto Alegre, Secretaria da Agricultura, Seção de Geografia, 1961. 4 2p.

PONS, S.B., MILAGRES J.C., TEIXEIRA, N.M. Efeitos de fatores genéticos e de ambiente sobre o crescimento e o escore de conformação em bovinos Hereford no Rio Grande do Sul. I - Peso e escore de conformação à desmama. Revista da Sociedade Brasileira de Zootecnia, Viçosa, v.18, n.5, p.391-401, 1989.

ROVIRA J. Manejo nutritivo de los rodeos de cria en partoreo. Montevideo : Hemirferio Sur, 1996. 288p.

SALOMONI, E., SILVEIRA, C.L.M. Acasalamento de outono em bovinos de corte: abrace essa idéias. Guaíba : Agropecuária, 1996. 152p.

SAS INSTITUTE INC. SAS/STAT. User' Guide, release 6.11 Edition. Cary : SAS Institute Inc, 1996. 1230p. 